\title{
The rotation of Io predicted by the Poincaré-Hough model
}

\author{
Benoît Noyelles ${ }^{1}$ \\ NAmur Center for Complex SYStems (NAXYS), University of Namur, Dpt of \\ Mathematics, 8 Rempart de la Vierge, B-5000 Namur, Belgium
}

\begin{abstract}
This note tackles the problem of the rotation of Io with the 4-degrees of freedom Poincaré-Hough model. Io is modeled as a 2-layer body, i.e. a triaxial fluid core and a rigid outer layer.

We show that the longitudinal librations should have an amplitude of about 30 arcseconds, independent of the composition of the core. We also estimate the tidal instability of the core, and show that should be slowly unstable.
\end{abstract}

Subject headings: Io - Resonances, spin-orbit - Rotational dynamics - Interiors - Celestial mechanics

\section{Introduction}

Thanks to the Galileo space mission in the Jovian system, we have now interesting clues on Io's internal structure. In particular, its mass and its second-order gravity field coefficients $J_{2}$ and $C_{22}$ are known with a good accuracy (Anderson et al. 2001). Moreover, internal heating due to an intense tidal dissipation (Peale et al. 1979; Lainey et al. 2009) is expected to induce a fluid core (Cassen et al. 1982).

We recently published a theoretical exploration of the Poincaré-Hough model applied to a synchronously rotating body (Novelles 2012). This model, originally proposed independently by Hough (1895) and Poincaré (1910) before being put in an Hamiltonian form by Touma \& Wisdom (2001), describes the rotational dynamics of a triaxial body composed of a rigid mantle and an ellipsoidal cavity filled by an inviscid fluid of constant uniform density and vorticity. This is a 4-degrees of freedom conservative model in which the core-mantle interactions result in pressure coupling at the core-mantle boundary.

\footnotetext{
${ }^{1}$ email: Benoit.Noyelles@fundp.ac.be, also associated with IMCCE, CNRS UMR 8028, Paris Observatory, UPMC, USTL, 77 avenue Denfert-Rochereau, 75014 Paris, France
} 
This paper proposes an application of this model to a realistic Io under the gravitational forcing of Jupiter. We build internal models and apply the Poincaré-Hough model to them, and we use complete orbital ephemerides, here from (Lainey et al. 2006), to model accurately the gravitational torque of Jupiter. After computation of Io's rotation we will discuss the problem of the elliptical instability of the fluid filling the core.

\section{The internal structure of Io}

We here consider Io to be an ellipsoidal body, its moments of inertia being $0<A \leq B \leq$ $C$. $A$ is the moment of inertia with respect to Io's long equatorial axis, while $C$ is related to the polar axis. The core is ellipsoidal as well, its principal axes of inertia being collinear to the ones of the whole body, the moments associated being written $A_{c}, B_{c}$ and $C_{c}$. The known gravity and shape parameters of Io are gathered in Tab.1.

Table 1: Gravity and shape parameters of Io. $I$ has not been measured independently of the others, it is deduced from the measured values of $J_{2}$ and $C_{22}$ combined with the hydrostatic equilibrium condition.

\begin{tabular}{l|r|r} 
Quantity & Value & Reference \\
\hline Mean density $\bar{\rho}$ & $3,527.8 \pm 2.9 \mathrm{~kg} / \mathrm{m}^{3}$ & Anderson et al. (2001) \\
$J_{2}$ & $(1.8459 \pm 0.0042) \times 10^{-3}$ & Anderson et al. (2001) \\
$C_{22}$ & $(5.537 \pm 0.012) \times 10^{-4}$ & Anderson et al. (2001) \\
$I /\left(M R^{2}\right)$ & $0.37685 \pm 0.00035$ & Anderson et al. (2001) \\
Mean radius R & $1,821.49 \mathrm{~km}$ & Archinal et al. (2011) \\
Subplanetary equatorial radius a & $1,829.4 \mathrm{~km}$ & Archinal et al. (2011) \\
Along orbit equatorial radius b & $1,819.4 \mathrm{~km}$ & Archinal et al. (2011) \\
Polar radius c & $1,815.7 \mathrm{~km}$ & Archinal et al. (2011)
\end{tabular}

The Poincaré-Hough rotational model requires 5 internal structure parameters:

- Polar flattening $\epsilon_{1}=\frac{2 C-A-B}{2 C}=J_{2} \frac{M R^{2}}{C}$,

- Equatorial ellipticity $\epsilon_{2}=\frac{B-A}{2 C}=2 C_{22} \frac{M R^{2}}{C}$,

- Polar flattening of the core $\epsilon_{3}=\frac{2 C_{c}-A_{c}-B_{c}}{2 C_{c}}$,

- Equatorial ellipticity of the core $\epsilon_{4}=\frac{B_{c}-A_{c}}{2 C_{c}}$,

- Relative inertia of the core $\delta=\frac{C_{c}}{C}$. 
From the definitions of $J_{2}=(2 C-A-B) /\left(2 M R^{2}\right)$ and the moment of inertia $I=$ $(A+B+C) / 3$, we have

$$
\frac{C}{M R^{2}}=\frac{I}{M R^{2}}+\frac{2}{3} J_{2}=0.37808 .
$$

We can see from these data that the 2 parameters $\epsilon_{1}$ and $\epsilon_{2}$ can be straightforwardly derived, yielding:

$$
\begin{aligned}
& \epsilon_{1}=4.88230 \times 10^{-3}, \\
& \epsilon_{2}=2.92901 \times 10^{-3} .
\end{aligned}
$$

The 3 parameters related to the size and shape of the core depend on its composition. We consider 2 end-members: either the core is made of pure iron, its density $\rho_{c}$ being $8,000 \mathrm{~kg} / \mathrm{m}^{3}$, or it is a eutectic mixture of FeS, yielding $\rho_{c}=5,150 \mathrm{~kg} / \mathrm{m}^{3}$ (Usselman 1975). Another uncertainty is in the composition of the crust. Anderson et al. (2001) considered 2 possibilities: either a thin $(\leq 50 \mathrm{~km})$ crust with a low density $\left(\leq 2,600 \mathrm{~kg} / \mathrm{m}^{3}\right)$, or a small amount of low density crust (that we propose to neglect) overlying a thicker (100$200 \mathrm{~km}$ ) melt-rich asthenosphere (density between 3,000 and $3,200 \mathrm{~kg} / \mathrm{m}^{3}$ ). The reader can find additional information on Io's internal structure in Moore et al. (2010). With these assumptions, we get 6 interior models of Io (Tab22).

Table 2: Physical parameters of our 6 models. The models 1,3 and 5 assume a pure iron core, while we have a eutectic FeS core in the models 2, 4 and 6 . The models 3 and 4 also consider a $30 \mathrm{~km}$-thick crust, while the models 5 and 6 have a $150 \mathrm{~km}$-thick crust. $\rho_{s}$ is the density of the crust, and $\rho_{m}$ the one of the mantle.

\begin{tabular}{r|rrrrrr} 
Models & $\begin{array}{r}\rho_{c} \\
\left(\mathrm{~kg} / \mathrm{m}^{3}\right)\end{array}$ & $\begin{array}{r}\rho_{m} \\
\left(\mathrm{~kg} / \mathrm{m}^{3}\right)\end{array}$ & $\begin{array}{r}\rho_{s} \\
\left(\mathrm{~kg} / \mathrm{m}^{3}\right)\end{array}$ & $\times 100$ & $\times 100$ & $\times 100$ \\
\hline 1 & 8,000 & 3,291 & - & 1.722 & 2.210 & 1.654 \\
3 & 8,000 & 3,377 & 2,400 & 1.836 & 2.673 & 1.270 \\
5 & 8,000 & 3,409 & 3,100 & 1.877 & 2.819 & 1.188 \\
\hline 2 & 5,150 & 3,243 & - & 0.980 & 1.057 & 6.503 \\
4 & 5,150 & 3,337 & 2,400 & 1.029 & 1.243 & 5.175 \\
6 & 5,150 & 3,351 & 3,100 & 1.033 & 1.253 & 5.160 \\
\hline
\end{tabular}

We can see that the 6 models can be splitted into 2 groups, the discrimination coming from the composition of the core. The equations used to derive these models can be found in Noyelles et al. (2011), Eq.1 to 12. 


\section{Rotational dynamics}

As already said, we use the Poincaré-Hough model to represent the rotational dynamics of Io. This model considers a rigid outer layer, composed of the mantle and eventually the crust, and a cavity filled by an inviscid fluid, constituting the fluid core. This cavity is triaxial, which allows pressure coupling at the core-mantle boundary. The computation of the rotational dynamics consists in numerically integrating the Hamilton equations derived from the following Hamiltonian:

$$
\mathcal{H}\left(p, P, r, R, \xi_{1}, \eta_{1}, \xi_{2}, \eta_{2}\right)=\mathcal{H}_{1}\left(P, \xi_{1}, \eta_{1}, \xi_{2}, \eta_{2}\right)+\mathcal{H}_{2}\left(p, P, r, R, \xi_{1}, \eta_{1}\right),
$$

where $\mathcal{H}_{1}$ is the kinetic energy of the system, and $\mathcal{H}_{2}$ the perturbing potential of Jupiter.

We have (see e.g. Noyelles (2012)) 


$$
\begin{aligned}
\mathcal{H}_{1}= & \frac{n}{2(1-\delta)}\left(P^{2}+\frac{P_{c}^{2}}{\delta}+2 \sqrt{\left(P-\frac{\xi_{1}^{2}+\eta_{1}^{2}}{4}\right)\left(P_{c}-\frac{\xi_{2}^{2}+\eta_{2}^{2}}{4}\right)}\left(\eta_{1} \eta_{2}-\xi_{1} \xi_{2}\right)\right. \\
& \left.+2\left(P-\frac{\xi_{1}^{2}+\eta_{1}^{2}}{2}\right)\left(\frac{\xi_{2}^{2}+\eta_{2}^{2}}{2}-P_{c}\right)\right) \\
+ & \frac{n \epsilon_{1}}{2(1-\delta)^{2}}\left(P_{c}^{2}-\left(\frac{\xi_{2}^{2}+\eta_{2}^{2}}{2}-P_{c}\right)^{2}+P^{2}-\left(P-\frac{\xi_{1}^{2}+\eta_{1}^{2}}{2}\right)^{2}\right. \\
+ & \left.2 \sqrt{\left(P-\frac{\xi_{1}^{2}+\eta_{1}^{2}}{4}\right)\left(P_{c}-\frac{\xi_{2}^{2}+\eta_{2}^{2}}{4}\right)}\left(\eta_{1} \eta_{2}-\xi_{1} \xi_{2}\right)\right) \\
+ & \frac{n \epsilon_{2}}{2(1-\delta)^{2}}\left(\frac{1}{4}\left(4 P-\xi_{1}^{2}-\eta_{1}^{2}\right)\left(\xi_{1}^{2}-\eta_{1}^{2}\right)+\frac{1}{4}\left(4 P_{c}-\xi_{2}^{2}-\eta_{2}^{2}\right)\left(\xi_{2}^{2}-\eta_{2}^{2}\right)\right. \\
- & \left.2 \sqrt{\left(P-\frac{\xi_{1}^{2}+\eta_{1}^{2}}{4}\right)\left(P_{c}-\frac{\xi_{2}^{2}+\eta_{2}^{2}}{4}\right)}\left(\eta_{1} \eta_{2}+\xi_{1} \xi_{2}\right)\right) \\
- & \frac{n \epsilon_{3}}{2(1-\delta)^{2}}\left(\delta\left(P^{2}-\left(P-\frac{\xi_{1}^{2}+\eta_{1}^{2}}{2}\right)^{2}\right)+\left(P_{c}^{2}-\left(\frac{\xi_{2}^{2}+\eta_{2}^{2}}{2}-P_{c}\right)^{2}\right)\left(2-\frac{1}{\delta}\right)\right. \\
+ & \left.2 \delta \sqrt{\left(P-\frac{\xi_{1}^{2}+\eta_{1}^{2}}{4}\right)\left(P_{c}-\frac{\xi_{2}^{2}+\eta_{2}^{2}}{4}\right)}\left(\eta_{1} \eta_{2}-\xi_{1} \xi_{2}\right)\right) \\
+ & \frac{n \epsilon_{4}}{2(1-\delta)^{2}}\left(\frac{\delta}{4}\left(4 P-\xi_{1}^{2}-\eta_{1}^{2}\right)\left(\eta_{1}^{2}-\xi_{1}^{2}\right)+\left(2-\frac{1}{\delta}\right) \frac{1}{4}\left(4 P_{c}-\xi_{2}^{2}-\eta_{2}^{2}\right)\left(\eta_{2}^{2}-\xi_{2}^{2}\right)\right. \\
+ & \left.2 \delta \sqrt{\left(P-\frac{\xi_{1}^{2}+\eta_{1}^{2}}{4}\right)\left(P_{c}-\frac{\xi_{2}^{2}+\eta_{2}^{2}}{4}\right)}\left(\eta_{1} \eta_{2}+\xi_{1} \xi_{2}\right)\right)
\end{aligned}
$$

and

$$
\mathcal{H}_{2}\left(p, P, r, R, \xi_{1}, \eta_{1}\right)=-\frac{3}{2} \frac{\mathcal{G} M_{4}}{n d^{3}}\left(\epsilon_{1}\left(x^{2}+y^{2}\right)+\epsilon_{2}\left(x^{2}-y^{2}\right)\right),
$$

where $\mathcal{G}$ is the gravitational constant, $M_{4}$ the mass of Jupiter, $n$ the orbital frequency of Io, and $d$ the distance Io-Jupiter. $x, y$ and $z$ are the coordinates of the vector pointing to Jupiter in the reference frame $\left(\vec{f}_{1}, \vec{f}_{2}, \vec{f}_{3}\right)$ linked to the principal axes of inertia of Io.

The canonical variables of the problem are: 


$$
\begin{array}{ll}
p, & P=\frac{G}{n C}, \\
r, & R=P(1-\cos K), \\
\xi_{1}=-\sqrt{2 P(1-\cos J)} \sin l, & \eta_{1}=\sqrt{2 P(1-\cos J)} \cos l \\
\xi_{2}=\sqrt{2 P_{c}\left(1+\cos J_{c}\right)} \sin l_{c}, & \eta_{2}=\sqrt{2 P_{c}\left(1+\cos J_{c}\right)} \cos l_{c} .
\end{array}
$$

Each of these 4 lines is related to a dynamical degree of freedom. The first one is related to the longitudinal motion of the whole body, $p$ is very close to the spin angle and $\vec{G}$ is the angular momentum of Io. $K$ is the obliquity with respect to the normal to the reference plane (here the equatorial plane of Jupiter at J2000.0), and $r$ is the node associated. The third degree of freedom is related to the polar motion (or wobble) of Io, $J$ being its amplitude. And the last one is related to the orientation of the velocity field of the fluid filling the core, we have in particular $P_{c}=G_{c} /(n C)$ where $\vec{G}_{c}$ is the angular momentum of the pseudo-core. The pseudo-core is very close to the core, it lacks of physical relevance but is convenient to write a Hamiltonian formulation of the equations. These canonical variables do not directly represent observables of the rotation (in fact only the surface can be observed), but exact observable quantities can be extracted from them.

The coordinates of Jupiter come from real ephemerides of Io, here L1.2 (Lainey et al. 2006). This way, we consider the orbital dynamics of Io with the most possible accuracy. The orbital period of Io is $\approx 1.769$ day and its eccentricity $4 \times 10^{-3}$. It experiences a $462-\mathrm{d}$ periodic perturbation due to the proximity of the $2: 1$ orbital resonance with Europa, and is locked into a laplacian orbital resonance involving also Europa and Ganymede.

The numerical integrations are performed with the 10th order Adams-Bashforth-Moulton predictor-corrector integrator. Once the solutions of the system have been obtained, we use Laskar's NAFF algorithm (Laskar 1993, 2005) to represent them as sums of quasiperiodic series, i.e.

$$
x(t) \approx \sum_{n=0}^{N} A_{n}^{\bullet} \exp \left(\imath \nu_{n}^{\bullet} t\right)
$$

for complex variables, or

$$
x(t) \approx \sum_{n=0}^{N} \mathcal{A}_{n}^{\bullet} \cos \left(\nu_{n}^{\bullet} t+\phi_{n}^{\bullet}\right)
$$

for real ones. In doing this, we can identify, in each variable of the problem, the influence of every single perturber (Jupiter, the other satellites, the Sun...).

Io is assumed to be in a dynamical equilibrium known as Cassini State 1 (Cassini 1693; 
Colombo 1966), as a consequence the initial conditions of the numerical integrations should be appropriately chosen. Deriving the equilibrium related to a simplified system (e.g. onedimensional rigid rotation and circular orbit of the perturber) is usually possible, but in a sophisticate system as we used, with complete ephemerides, it cannot be done accurately enough without using a perturbation theory. It is possible to derive an approximate equilibrium, but the numerical solutions will exhibit some free librations around the equilibrium, that are supposed to have been damped in the real system. For this reason, we chose to improve iteratively the initial conditions in using the algorithm by Noyelles et al. (2013), consisting in

1. starting from "pretty acceptable" initial conditions,

2. running a numerical integration,

3. identifying the free librations around the equilibrium,

4. removing them from the initial condions, and reiterate the process.

Once we have computed the equilibrum solution for our 6 models, we derive the following observable outputs:

- longitudinal librations of the mantle $\phi_{m}=p_{m}$ - nt, often called physical librations, where $p_{m}$ is the spin angle of the mantle,

- obliquity of the mantle $\epsilon_{m}$ (angle between the angular momentum of the mantle and the normal to the orbit of Io),

- amplitude of the polar motion $J_{m}$,

- tilt of the velocity field of the fluid $J_{c}$,

the formulae giving these quantities being extensively derived in Novelles et al. (2010). The results are gathered in Tab!3.

We can unfortunately see a high degeneracy in the sense that observing the rotation of Io should not allow to draw conclusions on its interior, the differences between the outputs being too small. The only number changing significantly is the tilt of the velocity field of the fluid $J_{c}$, that cannot be directly observed. 
Table 3: Variations of the outputs in the different models. $\phi_{m}$ is the amplitude of the longitudinal librations, $J_{m}$ the polar motion of the mantle, $J_{c}$ the tilt of the fluid, and $\epsilon_{m}$ is the obliquity of the mantle.

\begin{tabular}{|c|c|c|c|c|c|c|}
\hline Models & $\begin{array}{r}\phi_{m} \\
(462 \mathrm{~d}) \\
(\operatorname{arcsec})\end{array}$ & $\begin{array}{r}\phi_{m} \\
(1.76 \mathrm{~d}) \\
(\operatorname{arcsec})\end{array}$ & $<J_{m}>$ & $<J_{c}>$ & $<\epsilon_{m}>$ & $\begin{array}{r}\epsilon_{m} \\
(274 \mathrm{~d}) \\
(\operatorname{arcsec})\end{array}$ \\
\hline 1 & 39.82462 & 30.73077 & 136.592 & 5.89884 & 7.88443 & 2.35306 \\
\hline 3 & 39.82475 & 30.60912 & 135.689 & 5.52936 & 7.87640 & 2.33113 \\
\hline 5 & 39.82475 & 30.58332 & 138.148 & 5.41307 & 7.87537 & 2.32659 \\
\hline 2 & 39.82303 & 32.35491 & 139.912 & 15.82812 & 8.14896 & 3.60967 \\
\hline 4 & 39.82346 & 31.89343 & 140.550 & 13.13468 & 8.06166 & 3.08910 \\
\hline 6 & 39.82345 & 31.88809 & 136.018 & 12.78276 & 8.04716 & 3.07908 \\
\hline
\end{tabular}

\section{Elliptical instability}

In all the calculations, we have assumed that the flow of the fluid is laminar. In fact, as initially seen experimentally by Pierrehumbert (1986) and theoretically explained by Bayly (1986) in the context of an unbounced strained uniform vortex, the periodic forcing of the elliptical cavity on the underlying rotation state produces a pairwise resonance of inertial waves which can grow exponentially. These studies follow independent predictions in the 1970s, a recent review of the topic is given in Kerswell (2002). To check the stability of the flow we need to consider the growth rate $\sigma$ for an arbitrary perturbation $\vec{v}$, see e.g. Kerswell \& Malkus (1998):

$$
\sigma(t)=\frac{1}{2} \frac{d \ln <\vec{v}^{2} / 2>}{d t}=-\frac{<\vec{v} \cdot \vec{\nabla} U \cdot \vec{v}>}{\left\langle\vec{v}^{2}>\right.}
$$

where the flow $\vec{U}$ is the velocity field of the fluid a priori assumed to be laminar, and $<>=\int d V, V$ being the volume of the fluid. The flow is stable when $\sigma<0$, and unstable otherwise. Cebron et al. (2012) have recently derived the following formula:

$$
\sigma=n\left(\frac{17}{64} \epsilon \beta-2.62(1-\eta) \frac{1+\eta^{4}}{1-\eta^{5}} \sqrt{E}-\frac{\Lambda}{16}\right),
$$

in the context of a triaxial body perturbed by a primary whose rotation axis is close to the geometrical polar axis, and is under the influence of a magnetic field. The parameters involved in this formula are:

- $\epsilon$ : amplitude of the physical librations (Tab.3) at the orbital frequency (period: 1.76 
d)

- $\beta=\frac{a_{c}^{2}-b_{c}^{2}}{a_{c}^{2}+b_{c}^{2}}$

- $\eta$ : ratio between the internal and the external radii of the fluid layer. It is equal to 0 for fully liquid cores.

- Ekman number $E=\frac{\nu}{\Omega R_{c}^{2}}$ where $\nu$ is the fluid kinematic viscosity, and $\Omega=n P_{c} / \delta=n$ the mean velocity of the fluid. This is a kind of adimensional viscosity.

- Elsasser number $\Lambda=\frac{\sigma_{e} B_{0}^{2}}{\rho_{c} \Omega}$, where $B_{0}$ is the intensity of the magnetic field and $\sigma_{e}$ the fluid electrical conductivity. This contribution of the magnetic field has been derived by Cebron et al. (2012), generalizing a result by Herreman et al. (2009).

As Herreman et al. (2009) and Cebron et al. (2012) did, we took $B_{0}=1850 n T, \nu=$ $10^{-6} \mathrm{~m}^{2} \mathrm{~s}^{-1}$ and $\sigma_{e}=4 \times 10^{5} \mathrm{~S} . \mathrm{m}^{-1}$. The results are given in Tab.4.

Table 4: Grow rate $\sigma$, and the time associated compared with the spin-up time $t_{\text {spin-up. }}$.

\begin{tabular}{r|rrr} 
Models & $\begin{array}{r}\sigma \\
\left(\text { yr }^{-1}\right)\end{array}$ & $\begin{array}{r}1 / \sigma \\
(\text { kyr })\end{array}$ & $\begin{array}{r}t_{\text {spin-up }} \\
(\text { kyr })\end{array}$ \\
\hline 1 & $1.142 \times 10^{-3}$ & 0.875 & 3.321 \\
3 & $1.561 \times 10^{-3}$ & 0.640 & 3.140 \\
5 & $1.700 \times 10^{-3}$ & 0.588 & 3.109 \\
\hline 2 & $3.325 \times 10^{-4}$ & 3.008 & 4.770 \\
4 & $2.260 \times 10^{-4}$ & 4.425 & 4.559 \\
6 & $2.353 \times 10^{-4}$ & 4.250 & 4.557
\end{tabular}

The mean growth rate $\sigma$ is positive in every model. Anyway, it is smaller in the "even" models, i.e. with a $\mathrm{FeS}$ core, with a growth time of the order of 3,000 years, while it is always smaller than 1,000 years in the "odd" models (Fe core). For comparison we give in the last column the spin-up time $t_{\text {spin-up }}=1 /(n \sqrt{E})$ (Greenspan \& Howard 1963). This is the typical spin-up/spin-down time necessary for the fluid to recover the mantle velocity. $t_{\text {spin-up }}$ is usually assumed to be long enough so that the velocity of the fluid can be considered as constant. We can notice that it is of the same order of magnitude as the growth time $1 / \sigma$.

In all these models we find that the inertial waves of the core of Io should be unstable. The work of Kerswell \& Malkus (1998) showed that the waves are unstable, and Herreman et al. (2009) confirmed the result even when the effect of the magnetic field is included. This last reference suggests a growth time of $\approx 63$ years. However, Cebron et al. 
(2012) argue that this growth rate has been calculated in an extreme optimal case, in particular in considering an extremum of the instantaneous departure from the synchronous rotation. They conclude that the inertial waves in Io's fluid core should be stable. Our result lies between these two opposite conclusions, i.e. a positive but quite small growth rate. The main reason why our result is different from Cebron's with the same formula is that our $\beta$ (equatorial ellipticity of the core) is bigger. They also assumed a smaller amplitude of libration, derived by Comstock \& Bills (2003) for a rigid Io.

\section{Energy budget}

Io is known for its energy dissipation resulting in volcanos at its surface. Several attempts have been made to quantify this dissipation. One way to proceed is to use observations of Io's surface:

- Veeder et al. (1994) estimated the global heat flow $\phi$ to be bigger than $2.5 \mathrm{~W} / \mathrm{m}^{2}$,

- Matson et al. (2001) estimated it to be smaller than $13 \mathrm{~W} / \mathrm{m}^{2}$,

- Rathbun et al. (2004) estimated $\phi$ to be between 2.0 and $2.6 \mathrm{~W} / \mathrm{m}^{2}$, by studying several hot spots,

the reader can find additional references in Veeder et al. (2012), Tab.2. Another way is to try to detect the influence of tidal dissipation on the orbit of Io by comparing astrometric observations with dynamical models. Recently, Lainey et al. (2009) estimated the tidal dissipation to be $\dot{E}=(9.33 \pm 1.84) \times 10^{13} \mathrm{~W}$, yielding $\phi=2.24 \pm 0.45 \mathrm{~W} / \mathrm{m}^{2}$ assuming that energy is transported out of Io at the same rate.

The energy dissipation due to the instability of the fluid core is (Le Bars et al. 2010):

$$
\dot{E}=-\frac{8 \pi}{3} \rho_{c} R_{c}^{4} \sqrt{\nu}\left|\Omega^{s}-\Omega^{o}\right|^{5 / 2}\left|\omega_{S O}\right|^{5 / 2}
$$

where $M_{c}$ is the mass of the fluid core, $R_{c}$ its radius, $\rho_{c}$ its density, $\Omega^{s}=n P$ the spin rate of the mantle, and $\omega_{S O}$ is a normalized frequency of the spin-over mode, appearing in case of instability. We have $\omega_{S O}=\Omega^{f} / \Omega^{s}=1 / P$, since the spin rate of the fluid is assumed to be $n$.

Using formula (10) and $\left|\Omega^{s}-\Omega^{o}\right|=2 e|\cos n t|$ where $e \approx 0.004$ is the orbital eccentricity of Io, we get a mean $\dot{E}$ of $8.47 \times 10^{8} \mathrm{~W}$ for Model 1 and $2.32 \times 10^{9} \mathrm{~W}$ for Model 2. This 
is consistent with the conclusions of Le Bars et al. (2010) announcing a peak dissipation of $\approx 4 \times 10^{9} \mathrm{~W}$. This quantity is very small with respect to the energy dissipated by tides, i.e. $(9.33 \pm 1.84) \times 10^{13} \mathrm{~W}$.

Another possible source of dissipation is radioactive energy, because of the decay of unstable isotopes in the rock component of the body. Hussmann et al. (2010) estimate it between $3.08 \times 10^{11} \mathrm{~W}$ and $5.14 \times 10^{11} \mathrm{~W}$ depending on the composition of Io.

Table 5: Energy budget of Io, at the present time.

\begin{tabular}{lrr} 
Source of energy & $\dot{E}$ & Reference \\
\hline Surface heat flow & $8.33-10.83 \times 10^{13} \mathrm{~W}$ & Rathbun et al. $(\underline{2004})$ \\
Tidal dissipation & $(9.33 \pm 1.84) \times 10^{13} \mathrm{~W}$ & Lainev et al. $(2009)$ \\
Radioactive energy & $3.08-5.14 \times 10^{11} \mathrm{~W}$ & Hussmann et al. $(\underline{2010})$ \\
Fluid instability & $8.47 \times 10^{8}-2.32 \times 10^{9} \mathrm{~W}$ & this note
\end{tabular}

The results are gathered in Tab.5. We can see that if the flow of the fluid constituting the core of Io is unstable, the energy involved is negligible with respect to the tidal dissipation, as already stated Le Bars et al. (2010). So, this cannot be responsible for Io's volcanic activity.

\section{Conclusion}

In this study we used the Poincaré-Hough model to predict the rotation of Io, depending on its internal structure. For that, we elaborated 6 interior models, in considering pure iron or FeS compositions of the core, and the presence or not of a different crust. This rotational model describes the behavior of a 2-layer body composed of a rigid mantle and a fully liquid core, in considering pressure coupling at the core-mantle boundary.

For each case considered, the amplitude of the short longitudinal librations is about 30 arcsec, and the mean obliquity $\approx 8$ arcsec, these two quantities being a little bigger for a eutectic FeS core, which is larger. But the main difference is in the tilt of the angular momentum of the fluid constituting the core, that is bigger (between 10 and 15 arcsec) for the FeS than for the pure Fe one $(\approx 5$ arcsec). These differences should unfortunately not be detectable. A study of the elliptical instability indicates that the inertial waves should be unstable in any case, the growth time being between 500 and $800 \mathrm{yr}$ for a Fe core and between 3 and $5 \mathrm{kyr}$ for a FeS core. This last calculation only considers the influence of the longitudinal librations, without involving the tilt of the fluid. This instability does not have a significant impact on the energy dissipated at the surface. 


\section{Acknowledgements}

This research used resources of the Interuniversity Scientific Computing Facility located at the University of Namur, Belgium, which is supported by the F.R.S.-FNRS under convention No. 2.4617.07. The author is F.R.S.-FNRS post-doctoral research fellow, and is indebted to David Cébron for fruitful discussions.

\section{REFERENCES}

Anderson J.D., Jacobson R.A., Lau E.L., Moore W.B. \& Schubert G., 2001, Io's gravity field and interior structure, J. Geophys. Res., 106, 32,963-32,969

Archinal B.A., A'Hearn M.F., Bowell E., Conrad A., Consolmagno G.J., Courtin R., Fukushima T., Hestroffer D., Hilton J.L., Krasinsky G.A., Neumann G., Oberst J., Seidelmann P.K., Stooke P., Tholen D.J., Thomas P.C. \& Williams I.P., 2011, Report of the IAU Working Group on cartographic coordinates and Rotational Elements: 2009, Celestial Mechanics and Dynamical Astronomy, 109, 101-135

Bayly B.J., 1986, Three-dimensional instability of elliptical flow, Phys. Rev. Lett., 57, 21602163

Cassen P.M., Peale S.J. \& Reynolds R.T., 1982, Structure and thermal evolution of the Galilean satellites, in : Satellites of Jupiter, D. Morrison ed., 93-128, University of Arizona Press, Tucson

Cassini G.D., 1693, Traité de l'origine et du progrès de l'astronomie, Paris

Cebron D., Le Bars M., Moutou C. \& Le Gal P., 2012, Elliptical instability in terrestrial planets and moons, A\&A, 539, A78

Colombo G., 1966, Cassini's second and third laws, AJ, 71, 891-896

Comstock R.L. \& Bills B.G., 2003, A solar system survey of forced librations in longitude, J. Geophys. Res., 108(E09), 5100

Greenspan H.P. \& Howard L.N., 1963, On a time-dependent motion of a rotating fluid, Journal of Fluid Mechanics, 17, 385-404

Herreman W., Le Bars M. \& Le Gal P., 2009, On the effects of an imposed magnetic field on the elliptical instability in rotating spheroids, Physics of Fluids, 21, 046602 
Hough S.S., 1895, The oscillations of a rotating ellipsoidal shell containing fluid, Philosophical transactions of the Royal Society of London A, 186, 469-506

Hussmann H., Choblet G., Lainey V., Matson D.L., Sotin C., Tobie G. \& van Hoolst T., 2010, Implications of rotation, orbital states, energy sources, and heat transport for internal processes in icy satellites, Space Sci. Rev., 153, 317-348

Kerswell R.R. \& Malkus W.V.R., 1998, Tidal instability as the source for Io's magnetic signature, Geophys. Res. Lett., 25, 603-606

Kerswell R.R., 2002, Elliptical instability, Annual Review of Fluid Mechanics, 34, 83-113

Lainey V., Duriez L. \& Vienne A., 2006, Synthetic representation of the Galilean satellites' orbital motions from L1 ephemerides, A\&A, 456, 783-788

Lainey V., Arlot J.E., Karatekin Ö. \& Van Hoolst T., 2009, Strong tidal dissipation in Io and Jupiter from astrometric observations, Nature, 459, 957-959

Laskar J., 1993, Frequency analysis of a dynamical system, Celestial Mechanics and Dynamical Astronomy, 56, 191-196

Laskar J., 2005, Frequency map analysis and quasiperiodic decompositions, in Hamiltonian systems and fourier analysis: new prospects for gravitational dynamics, Benest et al. editors, Cambridge Sci. Publ., 99-129

Le Bars M., Lacaze L., Le Dizès S., Le Gal P. \& Rieutord M., 2010, Tidal instability in stellar and planetary binary systems, Physics of the Earth and Planetary Interiors, $178,48-55$

Matson D.L., Johnson T.V., Veeder G.J., Blaney D.L. \& Davies A.G., 2001, Upper bound on Io's heat flow, J. Geophys. Res., 106, 33021-33024

Moore W.B., Schubert G., Anderson J.D. \& Spencer J.R., 2010, The interior of Io, in Io after Galileo, A new view of Jupiter's volcanic Moon, Lopes R.M.C. \& Spencer J.R. eds., Springer Praxis, Chichester (UK)

Noyelles B., Dufey J. \& Lemaitre A., 2010, Core-mantle interactions for Mercury, MNRAS, 407, 479-496

Noyelles B., Karatekin Ö. \& Rambaux N., 2011, The rotation of Mimas, A\&A, 536, A61

Noyelles B., 2012, Behavior of nearby synchronous rotation of a Poincaré-Hough satellite at low eccentricity, Celestial Mechanics and Dynamical Astronomy, 112, 353-383 
Noyelles B., Delsate N. \& Carletti T., Equilibrium search algorithm of a perturbed quasiintegrable system, submitted, arXiv:1101.2138

Peale S.J., Cassen P. \& Reynolds R.T., 1979, Melting of Io by tidal dissipation, Science, 203, 892-894

Pierrehumbert R.T., 1986, Universal short-wave instability of two-dimensional eddies in an inviscid fluid, Phys. Rev. Lett., 57, 2157-2159

Poincaré H., 1910, Sur la précession des corps déformables, Bulletin Astronomique, 27, 321357

Rathbun J.A., Spencer J.R., Tamppari L.K., Martin T.Z., Barnard L. \& Travis L.D., 2004, Mapping of Io's thermal radiation by the Galileo photopolarimeter-radiometer (PPR) instrument, Icarus, 169, 127-139

Touma J. \& Wisdom J., 2001, Nonlinear core-mantle coupling, The Astronomical Journal, $122,1030-1050$

Usselman T.M., 1975, Experimental approach to the state of the core; Part I, The liquidus relations of the Fe-rich portion of the Fe-Ni-S system from 30 to $100 \mathrm{~kb}$, American Journal of Science, 275, 278-290

Veeder G.J., Matson D.L., Johnson T.V., Blaney D.L. \& Goguen J.D., 1994, Io's heat flow from infrared radiometry: 1983-1993, J. Geophys. Res., 99, 17095-17162

Veeder G.J., Davies A.G., Matson D.L., Johnson T.V., Williams D.A. \& Radebaugh J., 2012, Io: Volcanic thermal sources and global heat flow, Icarus, 219, 701-722 